\title{
Supervivencia de líquenes en Marte
}

\author{
Rosa DE LA TORRE NOETZEL \\ Dpto.Observación de la Tierra/Area de Investigación e Instrumentación Atmosférica/INTA (Instituto \\ Nacional de Técnica Aeroespacial), Crta. Ajalvir, km 4, 28850-Torrejón de Ardoz, Madrid \\ torrenr@inta.es
}

Recibido: $31 / 03 / 2016$

Aceptado: 13/09/2016

\section{Resumen}

Marte presenta en su superficie varias condiciones ambientales fundamentales, que condicionan la existencia de vida: la humedad, la presión, la composición de la atmósfera y la radiación. En la Tierra también existen áreas que climatológicamente son similares a Marte, expuestas a temperaturas muy bajas, a una extrema sequedad y a una alta radiación UV. Para estudiar la capacidad de supervivencia de organismos especialmente resistentes que viven en zonas análogas de Marte en la Tierra, los denominados extremófilos o extremotolerantes, se han usado cámaras climáticas que simulan parámetros climatológicos y la composición atmosférica de Marte. En éste estudio se muestran los resultados obtenidos con la especie de liquen extremófila y extremotolerante Circinaria gyrosa, que ha sido expuesta en PASC (Planetary Atmosferic Surface Chamber, INTA, CAB) a parámetros simulados de Marte, a una atmósfera rica en $\mathrm{CO}_{2}(95 \%)$, a una presión atmosférica de $7 \mathrm{mbar}$, a la radiación ultravioleta solar existente en la superficie de Marte $(>200 \mathrm{~nm})$ y a una temperatura de $-93^{\circ} \mathrm{C}$. Los parámetros fueron administrados en forma combinada e indeoendientemente, con el objetivo de obtener un conocimiento más profundo sobre los efectos, ya sea a nivel individual o todos a la vez. Los resultados obtenidos han mostrado una resistencia extremadamente alta de éste liquen a las condiciones ambientales simuladas de Marte.

Palabras clave: Simulación ambiental Marte; potencial de supervivencia; extremotolerante.

\section{Survival of lichens on Mars}

\begin{abstract}
On the surface of Mars several main aspects in combined form are conditioning the existence of life: temperature, humidity, pressure, composition of the atmosphere and radiation. The Earth also presents very dry and/or cold environments, which are close to Martian surface conditions with a high UV radiation. Due to the inability to test the survival capacity of extremely resistant organisms that live on these analog Mars environments, which are referred as extremophiles and extreme tolerant, climatic chambers that mimic the Mars climatology have been used. In this work we report the results obtained with the extreme tolerant lichen species Cicinaria gyrosa exposed at PASC (Planetary Atmospheric Surface Chamber, INTA, CAB) to Mars simulated parameters, that is, to a Mars atmospheric composition of mainly $95 \%$ of $\mathrm{CO} 2$ and a pressure of 7 mbar, to UV-solar radiation at Mars surface $(>200 \mathrm{~nm})$ and to a temperature of $-93{ }^{\circ} \mathrm{C}$, in combined- and independent form, in order to obtain a deeper understanding of the individual and joint effects. The results obtained have demonstrated an extremely high resistance of this lichen species against Mars simulated environmental conditions.
\end{abstract}

Key words: Mars environmental simulation; survival potential; extreme tolerant.

Sumario: Introducción. 1. Antecedentes 2. Trabajos realizados: Estudios de supervivencia de líquenes a la climatología de Marte 3. Metodología 4. Discusión y Resultados 5. Conclusiones. Agradecimientos. Referencias. 


\section{Referencia normalizada}

De la Torre Noetzel, R. (2016). Supervivencia de líquenes en Marte. Física de la Tierra, Vol., 28, 25-39.

\section{Introducción}

La investigación sobre la posibilidad de la existencia de vida pasada o presente en otros planetas no terrestres, es un tema muy actual que atrae el interés de la comunidad científica y público en general. Marte es uno de los planetas más investigados, teniendo en cuenta la existencia de agua investigada en el pasado (Horneck, 2000; McKay, 1997; Mehta et al., 2011 and Nelli et al., 2010) y sobre todo actualmente, en base al reciente descubrimiento de los restos minerales hidratados hallados en las laderas de los cráteres de Marte (Jim Green, 2015), por la sonda orbital Mars Reconnaissance Orbiter (MRO) de la NASA. Esto ha conducido a suponer la existencia de agua subterránea rica en sales que fluye hacia la superficie por medio de "arroyos" (RSL), dejando como huella sales hidratadas, en las estaciones más cálidas, y desapareciendo en las más frías, por lo que se cree que Marte podría incluso tener un ciclo geológico. Se abre de ésta forma, una nueva perspectiva en el estudio de la habitabilidad de Marte. Los datos de las sondas enviadas a Marte, desde 1965, han posibilitado un conocimiento más detallado del planeta, en cuanto a la climatología y a la composición mineralógica. Los parámetros ambientales en superficie, la elevada radiación, la composición y presión atmosférica, así como los extremos en temperatura, hacen que Marte sea un escenario extremo para la existencia de vida (Fig. 1). La evaluación de la resistencia de la vida a estas condiciones, implica averiguar primeramente los límites de la vida, es decir las condiciones fisicoquímicas más extremas a las que los organismos se pueden enfrentar, y en segundo lugar, descubrir cuáles son los mecanismos moleculares que permiten la supervivencia de estos organismos a esas condiciones extremas.

Actualmente la superficie de Marte presenta una temperatura media baja $\left(-50^{\circ} \mathrm{C}\right) \mathrm{y}$ es extremadamente seca excepto en los casquetes polares. La atmósfera es muy tenue (menor que un $1 \%$ de la presión atmosférica de la Tierra) y está formada principalmente por dióxido de carbono. Las misiones Viking permitieron observar que los minerales superficiales parecían ser oxidativamente muy activos, lo que produciría la descomposición rápida de compuestos orgánicos (McDonald et al., 1998). No se han encontrado compuestos orgánicos en Marte. Además la radiación solar ultravioleta intensa es destructiva para los compuestos orgánicos. Si Marte siempre hubiera presentado éste estado, sería improbable que la vida se hubiese desarrollado. Sin embargo, las observaciones del Mars Global Surveyor han mostrado imágenes de alta resolución de la superficie marciana con la existencia anterior de agua abundante: huellas de ríos fluyendo, de sedimentos, y de lechos de lagos, en las que algunas parecen recientes y pueden indicar la existencia de permafrost cerca de la superficie (McKay et al., 1996, Malin et al., 2000). Otras observaciones mineralógicas y magné- 
ticas apoyan la anterior presencia de una atmósfera de Marte más densa, con temperaturas más benignas y con más humedad, hace 3.8 billones de años.

La cuestión de vida posible en Marte (pasada o presente) está lejos de ser resuelta. Se han planificado programas de exploración de amplio alcance a nivel internacional al planeta rojo, que han llevado al desarrollo de cámaras ambientales de simulación de la climatología de Marte necesarias para la preparación e interpretación de dicha exploración planetaria (Brack et al., 1999). En ellas se han realizado estudios de caracterización de seres vivos muy simples, pero muy resistentes, que son capaces de sobrevivir a determinados factores climáticos extremos (Horneck et al., 2000).

Un organismo extremófilo es un organismo que vive en condiciones extremas, entendiéndose por tales aquellas que son muy diferentes a las que existente para la mayoría de las formas de vida en la Tierra. Se han identificado organismos extremófilos en zonas análogas a Marte en la Tierra, en la superficie y en la subsuperficie de la Antártida (Fig. 2) e Islandia, en desiertos fríos y calurosos (Atacama y Omán), y también en análogos del océano subsuperficial de hielo de la luna de Júpiter, "Europa", como pueden ser algunas zonas de la Antártida donde existen glaciares y lagos debajo del hielo superficial o fuentes hidrotermales.

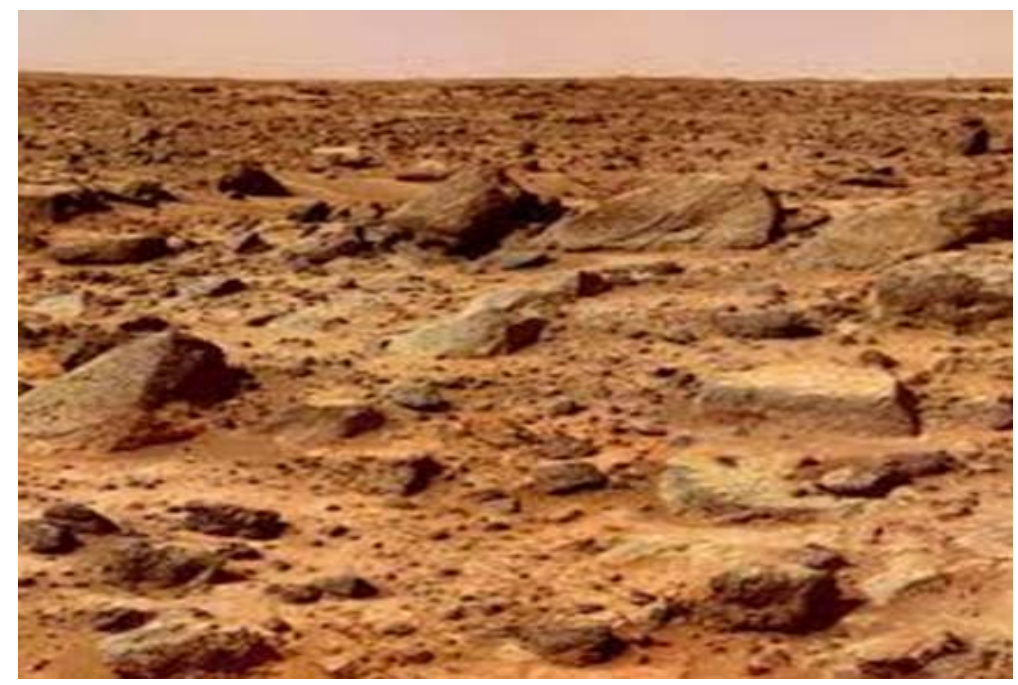

Figura 1. Foto representativa del paisaje superficial de Marte.

Estos organismos pueden ser procariotas o eucariotas, entre los que se han encontrado especies de líquenes muy resistentes a las condiciones de Marte. Los líquenes son organismos simbiontes formados por un alga y un hongo, en el que cada uno ejerce una función: el alga fotosintetiza y nutre al hongo, y éste protege al alga. Existen especies de líquenes que se consideran extremófilos debido a su resistencia a temperaturas extremas, radiación intensa y a la deshidratación. De allí que los líque- 
nes sean frecuentemente la forma dominante de vegetación en regiones polares y alpinas, y particularmente en zonas climáticas extremas, tales como los Valles Secos de la Antártida, donde constituyen la forma dominante de vida multicelular (Green et al., 2007). Estas regiones son comparables en base a ciertos parámetros climáticos con el espacio exterior: las temperaturas son extremas (largos períodos con temperaturas por debajo de cero), la radiación ultravioleta es muy elevada en verano y existen períodos de muy baja humedad relativa. Algunas especies de líquenes son capaces de colonizar estas áreas polares y regiones alpinas en los dos hemisferios. Experimentos de laboratorio han demostrado, que la capacidad de recuperación de su actividad metabólica es grande, incluso después de permanecer durante 10 años deshidratados y a temperaturas por debajo del punto de congelación y sin radiación solar, e incluso después de sumergirlos en nitrógeno líquido (Kappen, 2000; Kappen and Valladares, 2007). Por ello, los líquenes poseen una serie de propiedades que les permiten ser unos organismos idóneos para estudiar los límites de la vida en el espacio y en otros planetas.

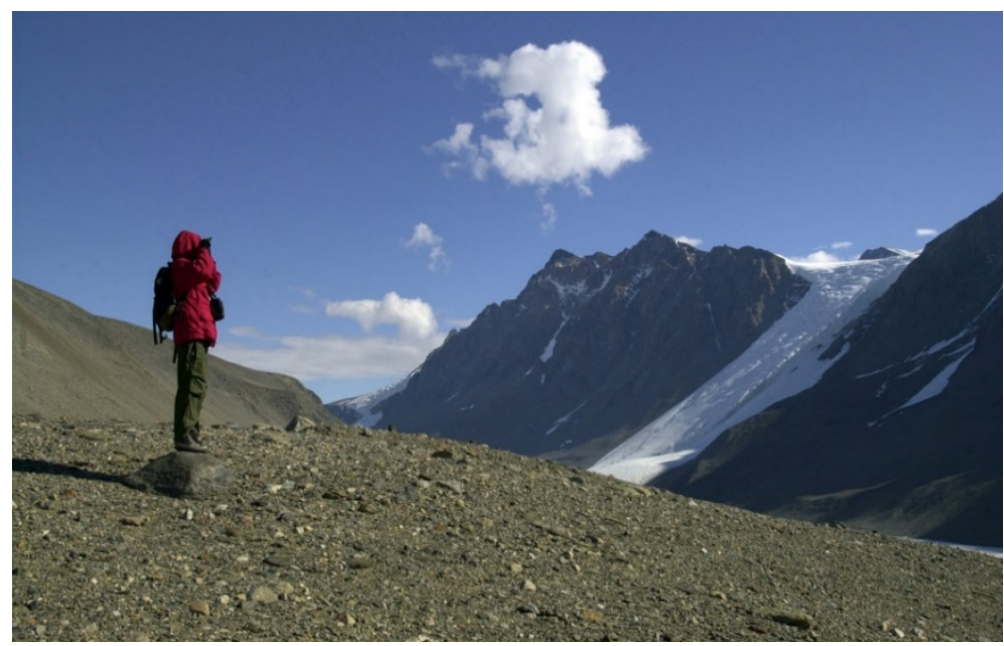

Figura 2. Taylor Valley (Antártida). Cortesía de Emily Stone/National Science Foundation.

\section{Antecedentes}

Se han realizado experimentos en el espacio con microorganismos (Horneck et al., 1993), con especies de líquenes extremófilos (Sancho et al., 2007) y con comunidades microbianas endolíticas (incluyendo líquenes), (De la Torre et al., 2010) que viven sobre y dentro de rocas, para estudiar la resistencia de estas especies en el espacio y en condiciones de Marte, además de comprobar su capacidad de resistir un hipotético viaje interplanetario, según postula la hipótesis de Litopanspermia (Panspermia, Arrhenius, 1903, Premio Nobel). Esta teoría, que apoya el origen de la vida entre diferentes planetas está basada en tres fases: un transporte interplanetario (Fase II) de 
organismos vivos, simples o poco evolucionados, tales como bacterias y cianobacterias o algas, incluidos en meteoritos, que pudieron haber sido expelidos de un planeta origen por medio de un impacto de una gran masa rocosa o meteoroide (Fase I). Los meteoritos resultantes portadores de vida habrían viajado por el espacio hasta un planeta destino, aterrizando sobre él (Fase III). Esta hipótesis podría ser aplicable a Marte y a la Tierra. Se han llevado a cabo varios experimentos a bordo del satélite Fotón, promovidos por la ESA (Agencia Espacial Europea) desde 2001, el primero fue LICHENS (Sancho et al., 2007) y el segundo fue LITHOPANSPERMIA (De la Torre et al., 2010), con el objetivo de demostrar que los líquenes y comunidades microbianas pueden sobrevivir en el espacio y considerarse potenciales propágulos interplanetarios de formas de vida.

LICHENS (Sancho et al., 2007) tuvo como objetivo estudiar, por primera vez, la capacidad de supervivencia de líquenes en el espacio, principalmente el efecto nocivo de la radiación UV extraterrestre, que incluye el espectro UV de Marte. Para ello se seleccionaron varias especies epilíticas en zonas de alta montaña (Sierra de Gredos y Sierra Nevada, 2000 m-2300 m a.s.1.), además de comunidades bacterianas y liquénicas endolíticas (interior roca) antárticas que fueron ubicadas dentro de un hardware experimental con dos niveles, uno superior para su exposición a la radiación extraterrestre y otro inferior por debajo del anterior o nivel control.

El experimento integrado en BIOPAN-5, plataforma experimental localizada en el exterior de la cápsula del satélite Foton M2 ruso, fue lanzado desde el cosmódromo de Baikonur a bordo de un cohete Soyuz en Mayo de 2005. Durante 16 días, las muestras fueron expuestas a radiación UV solar extraterrestre (UV-C $>170 \mathrm{~nm}$ ) y a un vacío espacial de $10^{-6} \mathrm{mB}$.Otro experimento relevante fue LITHOPANSPERMIA (Estudios de transferencia interplanetaria y de procesos de entrada de comunidades microbianas epi- y endolíticas, ESA- (ESA AO-2004-087, 2005)), (De la Torre et al., 2010), un experimento (Fig. 3a) para investigar, a un nivel más profundo, los mecanismos de resistencia de líquenes y también de comunidades microbianas (algas, bacterias, cianobacterias), en el espacio. El lanzamiento (Baikonur) fue en Septiembre de 2007, a bordo del satélite Foton M-3, permaneciendo en órbita (LEO) durante 10 días). Una vez de vuelta a la Tierra se realizaron análisis metabólicos y ultraestructurales, los primeros necesarios para registrar la actividad del PSII o rendimiento fotosintético (fotosistema II) a través de la fluorescencia de clorofila, y los siguientes para determinar el estado físiológico con microscopía confocal de láser (CLSM) y la ultraestructura a través de SEM (ScanningElectronMicroscopy) y LETM (LowElectron Transmision Microscopy). Sorprendentemente los resultados no mostraron el efecto nocivo de la radiación extraterrestre y del vacío espacial en combinación, tal como han mostrado muchos otros organismos, sino una recuperación de la actividad metabólica similar a la observada antes del vuelo. Esto nos condujo a identificar y definir, entre estas especies, al líquen Circinariagyrosa (Fig. 3b) como un sistemamodelo en exobiología, por la gran resistencia a nivel fisiológico y ultraestructural, debido a los mecanismos de protección altamente efectivos: un escudo protector o 
córtex (hongo) y a su naturaleza poiquilohídrica. De ésta forma los líquenes fueron incluidos dentro de los organismos más resistentes a la radiación UV, además de contribuir a la teoría de Litopanspermia. (De la Torre et al., 2010). Estos experimentos llevaron a plantear las siguientes conclusiones: los líquenes habían demostrado ser los primeros organismos simbiontes que eran capaces de sobrevivir en el espacio, lo cual era debido a la capacidad de entrar en un estado latente o de inactividad metabólica (anhidrobiosis o estado criptobiótico), característico de organismos que pueden tolerar la desecación extrema, como los líquenes (pérdida de hasta un 98\% de su agua corporal), cianobacterias, musgos y tardígrados (ositos de agua). De ésta forma los líquenes pasaban a formar parte de los organismos más resistentes a la radiación ultravioleta y al vacío espacial.

Además, los resultados de LITHOPANSPERMIA mostraron que no sólo eran capaces de recuperar su actividad metabólica prácticamente al 100\% después del vuelo, sino que también sus esporas eran capaces de germinar y de crecer (75-98\%), Este experimento contribuyó a esclarecer los procesos adaptativos y mecanismos de protección en hábitats extremos, además de ampliar los límites de la vida: los líquenes y las bacterias extremo tolerantes endolíticas son candidatos idóneos para mostrar que la vida es capaz de continuar después un hipotético viaje interplanetario, y además podrían sobrevivir a las condiciones en otros planetas habitables. Incluso la especie de cianobacteria Anabaenasp se había desarrollado después del vuelo en el laboratorio. Posteriores experimentos realizados con líquenes y comunidades criptoendolíticas (hongos, líquenes, etc.) sobre la ISS a largo plazo, tales como el experimento LIFE (Estudio de la capacidad de supervivencia de hongos líticos y líquenes en el espacio y en condiciones de Marte, integrado en la misión EXPOSE-E, ESA-NASA, sobre el Laboratorio Columbus, han mostrado que estos organismos no sólo son capaces de sobrevivir a condiciones reales de espacio durante más de 18 meses, sino también a condiciones de Marte (Onofri et al, 2012). Posteriormente (Onofri et al., 2012) se diseñó el experimento LIFE (LIchens and FungiExperiment), un experimento (Fig. 3c) con comunidades resistentes epilíticas (líquenes) y criptoendolíticas (hongos, algas, líquenes, que viven en el interior de rocas), fueron expuestas a largo plazo (año y medio, 2008-2009 misión EXPOSE-E, ESA-NASA) en el exterior del Laboratorio Columbusen la Estación Espacial Internacional (ISS). El objetivo era estudiar la probabilidad de supervivencia de éstos organismos en el espacio (vacío espacial, espectro completo de radiación solar UV extraterrestre $(>110 \mathrm{~nm})$ y radiación cósmica), y también en condiciones superficiales de Marte ( $>200 \mathrm{~nm}$, presión y composición atmosférica de Marte). Los resultados después del vuelo mostraron que estos organismos extremófilos terrestres resisten las condiciones de espacio real y también las de Marte simuladas durante 18 meses (Onofri et al., 2012). Estos resultados son relevantes en cuanto a que ayudan a determinar la protección óptima frente a la radiación, además de ayudar a identificar sistemas fotosintéticos resistentes a la radiación UV, que puedan ser aplicables en investigación básica enfocados en la producción de oxígeno, fuel, biomasa y nutrientes y biomining (extracción de minerales en rocas o suelos a través de bacterias), fundamentalmente para viajes espaciales largos. Para 
llevar a cabo todos estos experimentos en el espacio, la ESA ha exigido realizar ensayos de simulación ambientales, que simulan el ambiente espacial. Por ello todos los organismos fueron sometidos previamente a condiciones de espacio y Marte con el fín de comprobar su resistencia antes del vuelo. Los estudios se realizaron en hardwares, desarrollados por el INTA (Área Atmósfera y Cargas Útiles), y siendo sometidos a vacío espacial $\left(10^{-5} \mathrm{~Pa}\right)$, composición atmósfera y presión $\left(10^{3} \mathrm{~Pa}\right)$ de Marte, ciclos de temperatura extrema, y radiación UV extraterrestre $(200-400 \mathrm{~nm})$, de forma combinada e independiente, en las cámaras de simulación del INTA (Spasolab/CAB), del DLR Colonia (Instituto de Medicina Aeroespacial) y del DLR-Berlín (Instituto de Planetología).
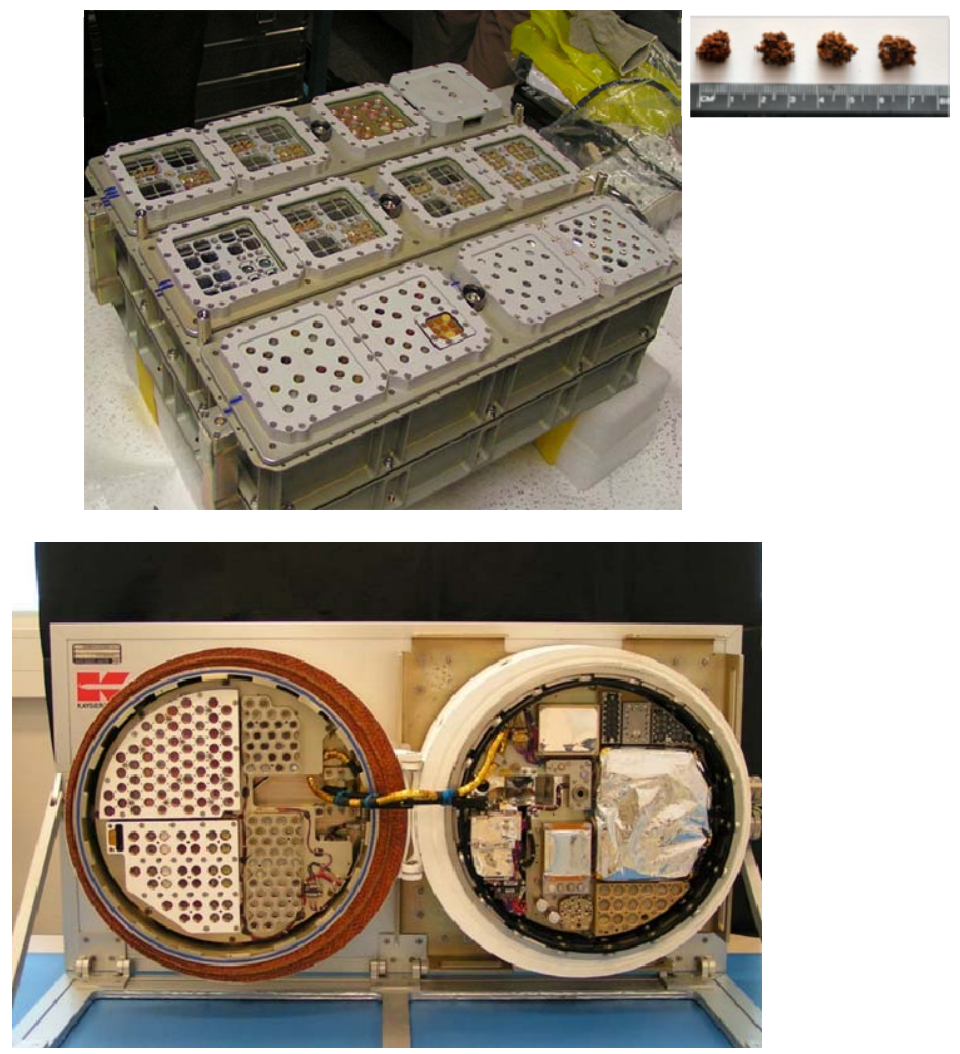

Figura 3. Experimento LITHOPANSPERMIA: a) paquete experimental integrado en BIOPAN-6 (Misión Fotón M-3, 2007, foto cortesía de ESA); 3b) muestras de la especie de liquen C. gyrosa. (Foto cortesía de U. Deutschmann); 3c) EXPOSE-E incluyendo el experimento LIFE (Foto cortesía de ESA). 


\section{Trabajos realizados: Estudios de supervivencia de líquenes a la climatología de Marte}

Las misiones espaciales llevadas a cabo hasta ahora han posibilitado un mejor conocimiento de las condiciones ambientales de planetas, entre los que se sitúa Marte. Investigaciones realizadas, muestran a Marte como un planeta con condiciones hostiles para el desarrollo de la vida, principalmente sobre su superficie, ya que está expuesta a una elevada radiación ultravioleta $\mathrm{C}$, muy dañina para seres vivos, y una composición atmosférica, altamente tóxica por elevado contenido en $\mathrm{CO} 2$ de un $95 \%$ con un 5\% restante de nitrógeno y argón y menos del $0,2 \%$ de oxígeno y vapor de agua. Además la presión atmosférica es más de 100 veces menor que sobre la Tierra $(6,1 \mathrm{mB})$, y las temperaturas son extremas, desde $-93^{\circ} \mathrm{C}$ hasta $+26^{\circ} \mathrm{C}$.

Para estudiar los efectos a los parámetros de Marte, en combinación o en forma aislada, en organismos extremófilos, se han realizado experimentos de simulación de condiciones marcianas en cámaras de simulación planetaria, tales como PASC (Planetary Atmospheric Simulation Chamber) (Fig.4) en el INTA/CAB y en el DLR (Centro Aeroespacial Alemán). Debido a las limitaciones técnicas y económicas obvias para la exploración planetaria in-situ, los experimentos en cámaras de simulación son las opciones más factibles para reproducir el ambiente de un planeta (Galletta et al., 2006; Jensen et al., 2008; Mateo-Martí et al. 2006; Zhukova et al., 1965 and Zill et al., 1979), ya que posibilitan el control de los parámetros, protocolos de análisis, además de la medida con sensores (Garry et al., 2006; Nicholson and Schuerger, 2005; Sears et al., 2002 and Smith et al., 2009), permitiendo la exposición de muestras a condiciones aisladas de Marte o conjuntas. Se han realizado experimentos con gran variedad de organismos extremófilos para estudiar la capacidad de supervivencia a condiciones simuladas de Marte, mostrando una cierta resistencia a algunos parámetros climáticos. Entre los más resistentes se encuentra, Circinariagyrosa, liquen vagante (Sancho et al., 2000) recolectada en la meseta central ibérica (Provincia de Guadalajara), caracterizada por una óptima adaptación a condiciones ambientales de hábitats áridos y semiáridos, y a fluctuaciones en las temperaturas diurnas y estacionales (desde $38,0^{\circ} \mathrm{C}$ a $-8,2^{\circ} \mathrm{C}$ ), aparte de ser capaz de resistir una sequedad extrema y elevados niveles de radiación solar (Sancho et al., 2000). Su supervivencia después de 10 días en el espacio a bordo del satélite Fotón (Experimento Lithopanspermia, 2007) ha llevado a seleccionarla como una especie idónea para estudiar los límites de la vida, en base a su estudio en condiciones simuladas de Marte. Para ello se realizaron tests de simulación en una cámara ambiental existente en el Instituto de Astrobiología del INTA, PASC (Planetary Atmospheric Simulation Chamber). La elevada resistencia mostrada por algunas especies de líquenes ha permitido identificarlos como organismos modelo en astrobiología. 


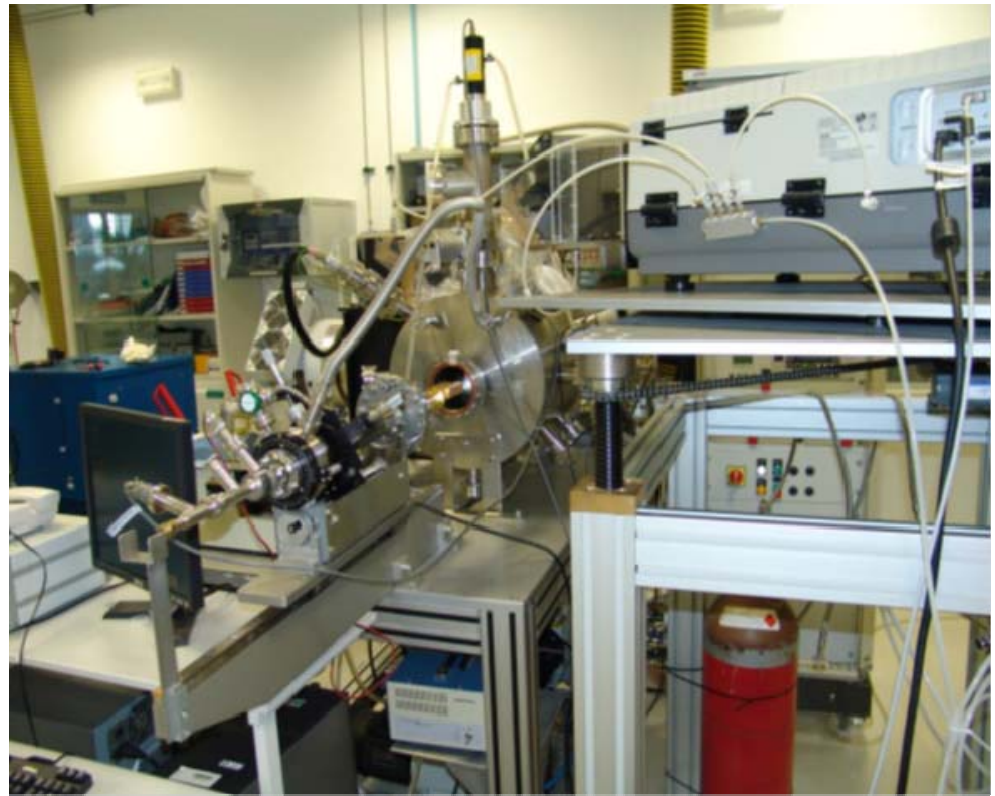

Figura 4. PASC (Planetary Atmospheres Simulation Chamber).

\section{Metodología}

Los parámetros simulados fueron:

- Atmósfera: La composición de gases se dio en las proporciones: $95 \% \mathrm{CO}_{2}$, $2,7 \% \mathrm{~N}_{2}, 1,6 \%$ Ar y $0,6 \% \mathrm{H}_{2} \mathrm{O}$, de acuerdo con Owen (1992).

- Presión: La presión global dentro de la cámara se midió en 7 mbar. En un tratamiento se aplicó un vacío de $10^{6} \mathrm{mbar}$, el presente en LEO.

- Temperatura: Se estableció una temperatura de $-93^{\circ} \mathrm{C}$, siendo ésta la mínima temperatura esperada que se alcanza en los sitios de amartizaje de los rovers enviados a Marte y es al mismo tiempo su límite para operaciones técnicas. Se llevaron a cabo tres combinaciones de parámetros ambientales marcianos (y de vacío espacial) simulados. Estos se aplicaron sobre muestras de C. gyrosa dispuestas en dos niveles ( 1 y 2). Las combinaciones de parámetros reproducidos en cada una de esas simulaciones y los objetivos década combinación de condiciones fueron las siguientes:

- Tratamiento 1: Atmósfera, presión y temperatura $\left(-93^{\circ} \mathrm{C}\right)$ marcianas simuladas. 
Objetivo: Detectar diferencias entre el nivel I y nivel II del porta muestras no debidas a la radiación $\mathrm{UV}$.

- Tratamiento 2: Atmósfera, presión, temperatura $\left(-93^{\circ} \mathrm{C}\right)$ y radiación UV $(200-400 \mathrm{~nm})$ marcianas simuladas.

Objetivo: Estudiar y cuantificar el efecto de la radiación UV sobre las muestras.

- Tratamiento 3: Vacío LEO, temperatura $\left(-93^{\circ} \mathrm{C}\right)$ y radiación UV marciana simulada.

Objetivo: Estudiar y cuantificar el efecto de la combinación de los dos parámetros espaciales más letales: la radiación UV-C y el vacío sobre C. gyrosa.

\section{Discusión y Resultados}

La influencia de los parámetros tales como la presión, la composición de gas y la radiación UV sobre la actividad biológica de los líquenes se estudió por medio de dos distintos sistemas. Después de la simulación y de la consiguiente reactivación de las muestras se realizaron medidas del rendimiento máximo cuántico (Yield) del fotosistema II (MQY) y del intercambio gaseoso. Los valores de rendimiento fotosintético no revelaron diferencias significativas en ninguno de los tratamientos experimentales aplicados en comparación con las muestras control (medias MQY 1/40.5670.03 con n1/429 AOI). En el tratamiento 1 (Fig. 5a), las muestras del nivel inferior 2 muestran una disminución insignificante en MQY con respecto de un 0.6170.07-0.5970.10 en las medidas de exposición pre- y post, respectivamente. También las muestras del nivel superior 1 muestran una disminución insignificante $(0.6070 .05-0.5370 .12)$ en las medidas antes y después de la exposición. En el tratamiento 2 (Fig. 5b), las muestras del nivel 2 (controles oscuros) mostraron un incremento insignificante de MQY (0.5170.14-0.6070.05), mientras las muestras irradiadas del nivel 1no exhibían cambios $(0.5470 .10-0.5470 .10)$. En el tratamiento 3, que estudiaba el efecto combinado de radiación UV simulada de Marte, de temperatura baja y de vacío LEO (1×10 mbar), sobre el MQY delas muestras de C. gyrosa, las medidas del nivel 2 y del nivel 1 , antes y después de su exposición, también mostraban disminuciones insignificantes y mínimas en MQY (0.6370.07 - 0.6170.04 y de 0.6170 .05 a 0.5970.05). La comparación de estos valores con los de las muestras no-tratadas, y con las muestras de los diferentes tests no mostró diferencias significativas, tanto en los valores antes, como de después de la exposición de MQY. Estos resultados conducen a las siguientes conclusiones: se ha demostrado la comparabilidad entre las muestras del nivel superior, como el inferior. Las tres combinaciones diferentes de los parámetros simulados de Marte no tienen un efecto significativo sobre el MQY de C. gyrosa. Comparando los resultados obtenidos en el tratamiento 1 con el tratamiento 2 , y del tratamiento 2 con el tratamiento 3 se puede concluir que la radiación UV y el vacío LEO en forma aislada, no tienen efecto sobre el MQY del liquen. 

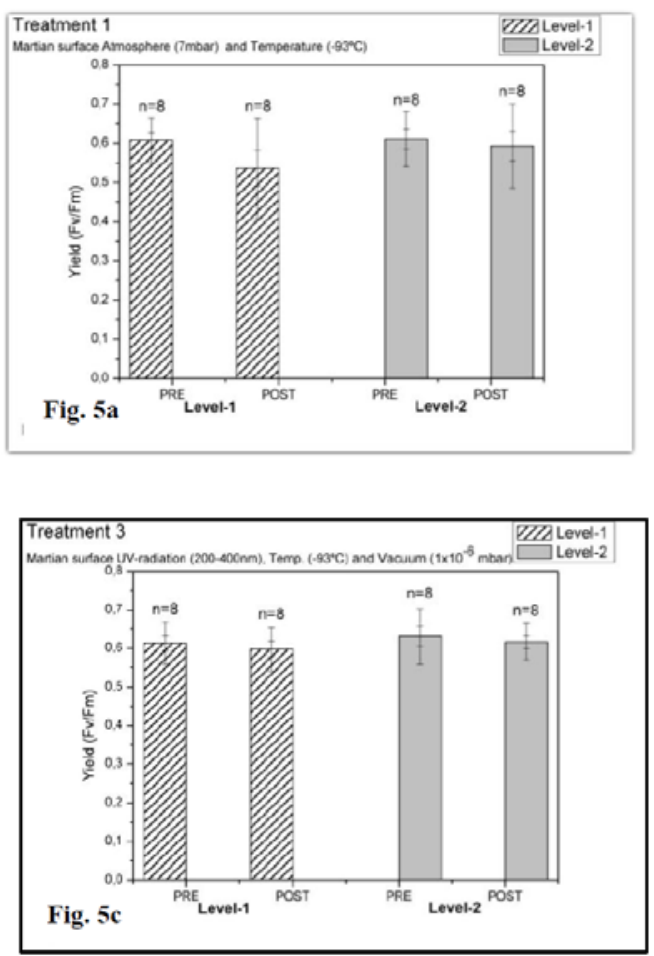

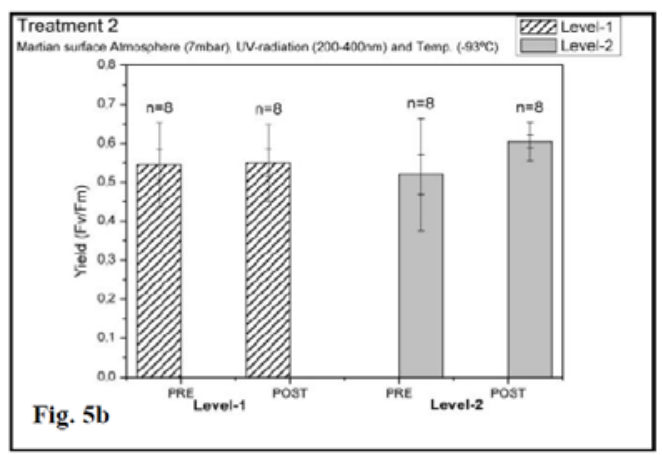

Figura 5. Rendimiento fotosintético en valores de yield máximo quántico (MQY) que muestran las muestras del nivel 1 superior y las muestras del nivel 2 inferior antes y después de la simulación: 5a) Marte (atmósfera, presión y temperatura); 5b) Marte (atmósfera, presión, temperatura y radiación UV); $5 \mathrm{c}$ ) vacio $\mathrm{LEO}$, temperatura $\left(-93^{\circ} \mathrm{C}\right)$ y radiación UV marciana simulada.

\section{Conclusiones}

Resultados de estudios previos realizados con otras especies de líquenes extremófilas sometidas a condiciones de Marte (atmósfera, temperatura y humedad) realizados durante 22 días, no han demostrado una disminución significativa en la vitalidad del organismo estudiado (de Vera et al., 2010a). Estos datos, junto con los resultados precedentes, que muestran unos niveles extraordinarios de resistencia a los parámetros ambientales de espacio y de ambientes planetarios (de la Torre et al., 2010; de Vera et al., 2010a, 2004a, 2003; de Vera and Ott, 2010; Onofri et al., 2012; Raggioet al., 2011; Sancho et al., 2009, 2007), han llevado a considerar que la capacidad de los líquenes - como organismos poiquilohídricos - de sobrevivir a condiciones ambientales inapropiadas, podría ser debido a una adaptación más generalizada de los líquenes. Esto se traduce en la capacidad de pasar a un estado ametabólico, llamado anabiosis, o criptobiosis más específicamente.

Este aspecto junto con su estructura interna, podría explicar la resistencia de éste liquen a las condiciones de espacio y de Marte. Esto sugiere que $\mathrm{C}$. gyrosa es un buen modelo sistemático, para estudios futuros relacionados con la astrobiología. Además éste estudio, en combinación con las altas tasas de supervivencia a condiciones reales 
de LEO detectadas después del experimento espacial LITHOPANSPERMIA (de la Torre et al., 2010), hace que la hipótesis de Litopanspermia (transferencia de organismos por medio de meteoritos) cobre más importancia.

Además, teniendo en cuenta la relevancia de cámaras de simulación planetaria que hace que sea una instrumentación esencial para emular las condiciones planetarias, el estudio de la habitabilidad de organismos biológicos al ambiente de Marte ha sido demostrado con éste estudio.

\section{Agradecimientos}

La autora quiere expresar su agradecimiento a Francisco Javier Sánchez-Iñigo, por su excelente aportación profesional a los experimentos realizados en el INTA, a Mercedes Vivas (colaboración en análisis estadístico), a Manuel Reina Aranda por su apoyo técnico, a J. A. Martín-Gago por su soporte en PASC, a J. M. Sobrado por la manutención técnica de PASC y a Gerda Horneck por su continuo soporte científico. Los experimentos de simulación de Marte se llevaron a cabo en el Centro de Astrobiología (CAB) y fueron subvencionados por la Unión Europea, por el Instituto Nacional de Técnica Aeroespacial-INTA y por el Ministerio de Ciencia e Innovación (AYA2010-11422-E (SubprogramaESP), CGL2006-12179-C02-01 y CTM200912838-C04-01). Los estudios anatómico-morfológicos fueron financiados por el DLR (German Aerospace Center) en el marco del experimento de la ESA-space experiment BIOMEX (ILSRA ESA-ILSRA 2009-0834, P-I Dr. Jean P. de Vera).

\section{Referencias}

ARRHENIUS, S. (1903). Die Verbreitung des LebensimWeltenraum. Umschau 7: 481-485.

BÖTTCHER, U., MEESSEN, J., MARTINEZ-FRÍAS, J., HÜBERS, H.-W., RULL, F., SÁNCHEZ, F.J., DE LA TORRE, R., DE VERA, J.-P. (2013). Raman Spectroscopic Analysis of the Calcium Oxalate Producing Extremotolerant Lichen Circinariagyrosa. International Journal of Astrobiology 00 (2013) 1-14.

BRACK, A., CLANCY, P., HOFMANN, B., HORNECK, G., KURAT, G., MAXWELL, J., ORI, G.,

PILLINGER, C., RAULIN, F., THOMAS, N., WESTALL, F. (1999). An integrated exobiology package for the search for life on Mars. Advances in Space Research, 23 (2), 301-308.

DE LA TORRE, R., SANCHO, L.G., HORNECK, G., DE LOS RÍOS, A., WIERZCHOS, J., OLSSON-

FRANCIS, K., COCKELL, C., RETTBERG, P., BERGER, T., DE VERA, J.P., OTT, S., FRÍAS, J. M., GONZÁLEZ, P.M., LUCA, M.M., REINA, M., PINTADO, A., DEMETS, R. (2010). Survival of lichens and bacteria exposed to outer space conditions - Results of the Lithopanspermia experiments, Icarus 208, 735-748. 
DE VERA, J.-P., HORNECK, G., RETTBERG, P., OTT, S. (2003). The potential of the lichen symbiosis to cope with the extreme conditions of outer space I. Influence of UVradiation and space vacuum on the vitality of lichen symbiosis and germinationcapacity. Int. J. Astrobiol. 1, 285-293.

DE VERA, J.-P., HORNECK, G., RETTBERG, P., OTT, S. (2004a). The potential of the lichen symbiosis to cope with the extreme conditions of outer space II: germination capacity oflichen ascospores in response to simulated space conditions. Adv. Space Res. 33, 1236-1243.

DE VERA, J.-P., MÖHLMANN, D., BUTINA, F., LOREK, A., WERNECK, R., OTT, S. (2010a). Survival potential and photosynthetic activity of lichens under Mars-like conditions: alaboratory study. Astrobiology 10 (2), 215-227.

DE VERA, J.-P., OTT, S. (2010b). Resistance of Symbiotic Eukaryotes. Survival to SimulatedSpace Conditions and Asteroid Impact Cataclysms. In "Symbioses and Stress: Joint Ventures in Biology" Cellular Origin, Life in Extreme Habitats and Astrobiology J. Seckbach and M. Grube (Eds.), 17:595-611.

GALLETA, G., FERRI, F., FANTI, G., D'ALESSANDRO, M., BERTOLONI, G., PAVARIN, D., BETTANINI, C., COZZA, P., PRETTO, P., BIANCHINI, G., DEBEI, S. (2006). S.A.M., the Italian Martian Simulation Chamber Origins of Life and Evolution of the Biosph. 36/5-6, 625-627.

GARRY, J.R.C. TEN-KATE, I.L. MARTINS, Z. NORNBERG, P. EHRENFREUD, P. (2006). Analysis and survival of amino acids in Martian regolith analogs Meteoritics and Planetary Science 41-3, 391-405.

GREEN, TGA, SCHROETER, B, SANCHO, LG. (2007). Plant life in Antarctica. In: Pugnaire F, Valladares F (eds), Functional Plant Ecology. CRC Press, Boca Raton, Florida, pp. 389-433.

GREEN, T.G.A. (2009). Lichens in arctic, antarctic and alpine ecosystems. In: Ökologische Rolle der Flechten Rundgespräche der Kommissionfür Ökologie 36. BayerischeAkademie der Wissenschaften (Herausgeber), Verlag Dr. Friedrich Pfeil, München, pp 45-65.

HORNECK, G., 1993. Responses of Bacillus subtilis Spores to Space Environment: Results from Experiments in Space, Origins of Life 23, 37-52.

HORNECK, G. (2000). The microbial world and the case for Mars. Planet. and Space Sci.25 48 (11), 1053-1063.

JENSEN, L.L., MERRISON, J., HANSEN, A.A., MIKKELSEN, K.A., KRISTOFFERSEN, T., NORNBERG, P., LOMSTEIN, B.A., FINSTER, K. (2008). A Facility for Long-Term Mars Simulation Experiments: The Mars Environmental Simulation Chamber (MESCH) Astrobiology 8-3, 537-548.

KAPPEN, L. (2000). Some aspects of the great success of lichens in the Antarctica. Antarctic Science 12: 314-324.

KAPPEN, L., VALLADARES, F. (2007). Opportunistic growth and desiccation tolerance, the ecological success ofpoikilohydrous autotrophs. In: Pugnaire F, Val- 
ladares F (eds), Functional Plant Ecology. CRC Press, Boca Raton, Florida, pp. 765.

MATEO-MARTÍ, E., PRIETO-BALLESTEROS, O., SOBRADO, J. M., GÓMEZELVIRA, J., MARTÍN-GAGO, J. A. (2006). A chamber for studying planetary environments and its applications to astrobiology Measurement and Science Technology 17, 2274-2280.

McDONALD, G. D. (1998). Icarus 132 (1), 170-5.

McKAY, C. P. (1997). The search for life on Mars. Orig. Life Evo. Bioph. 27, 263289.

MEHTA, M., RENNO, N.O., MARSHALL, J., GROVER, M.R., SENGUPTA, A., RUSCHE, N.A., KOK, J.F., ARVIDSON, R.E., MARKIEWICZ, W.J., LEMMON, M., SMITH. P.H. (2011). Explosive erosion exposesthe subsurface ice on Mars. Icarus, 211, 172- 194.

NELLI, S.M., RENNO, N.O., MURPHY, J.R., FELDMAN, W.C. (2010). Simulations of Atmospheric

Phenomena at the Phoenix Landing Site with the Ames GCM. Journal of Geophysical Research, Special Issue on Phoenix, 115, E00E21.

NICHOLSON, W.L., SCHUERGER, A.C. (2005). Bacillus subtilis Spore Survival and Expression of Germination-Induced Bioluminescence After Prolonged Incubation Under Simulated Mars Atmospheric Pressure and Composition: Implications for Planetary Protection and Lithopanspermia Astrobiology 5-4, 536-544.

ONOFRI, S., DE LA TORRE, R., DE VERA, J.P., OTT, S., ZUCCONI, L., SELBMANN, L., SCALZI, G.VENKATESWARAN, K.J., RABBOW, E., SÁNCHEZ, F.J., HORNECK, G. (2012). Survival of rock-colonizing organisms after 1.5 year in outer space. Astrobiology 12 (5), 508-516.

OWEN, T.C. (1992). The composition and early history of the atmosphere of Mars. Mars,University of Arizona Press, Tucson.

RAGGIO, J., PINTADO, A., ASCASO, C., DE LA TORRE, R., DE LOS RÍOS, A., WIERZCHOS, J., SANCHO, L.G. (2011). Whole Lichen Thalli Survive Exposure to Space Conditions: ResultsOf Lithopanspermia Experiment with Aspiciliafruticulosa. Astrobiology, 11 (4), 281-292.

SANCHO, LG, SCHROETER, B, DEL PRADO, R. (2000). Ecophysiology and morphology of the globular erratic lichen Aspicilia fruticulosa from Central Spain. Bibliotheca Lichenologica 75:137-147.

SANCHO, L.G., DE LA TORRE, R., HORNECK, G., ASCASO, C., DE LOS RÍOS, A., PINTADO, A., WIERZCHOS, J., SCHUSTER, M. (2007). Lichens survive in space: Results from 2005 LICHENS experiment. Astrobiology 7, 443-454.

SANCHO, L.G., DE LA TORRE, R., PINTADO, A. (2009). Lichens, new and promising material from experiments in astrobiology. Fungal Biology Reviews (homepage: www.elsevier.com/locate/fbr).

SEARS, D.W.G., BENOIT, P.H., McKEEVER, S.W.S., BANERJEE, D., KRAL, T., SUITES, W., ROE, L., JANSMA, P., MATTIOLI, G. (2002). Investigation of bio- 
logical, chemical and physical processes on and in planetary surfaces by laboratory simulation. Planetary and Space Science 50-9, 821-828.

SMITH, D. J., SCHUERGER, A.C., DAVIDSON, M.M., PACALA, S.W., BAKERMANS, C., ONSTOTT, T.C. (2009). Survivability of PsychrobactercryohalolentisK5 under Simulated Martian Surface Conditions. Astrobiology 9-2, 221228.

ZILL, L.P., MACK, R., DEVINCENZI, D.L. (1979). Mars ultraviolet simulation facility. Journal of Mol. Evolution 14, 1-3, 79-89

ZHUKOVA, A.I., KONGRATYEV, I., ONDRATYEV, I.I. (1965). On artificial Martian conditions reproduced for microbiological research. Life Sci. Space Res. 3. 120-126.

\section{INFORMACIÓN ADICIONAL}

http://www.rtve.es/alacarta/videos/television/acuerdas---orgenes-estacion-espacialinternacional/948163/

http://www.nasa.gov/multimedia/nasatv/\#.U8z4U_5Dt0x

http://www.spacenewsfeed.com/index.php/news/1575-survivalists-ready-for-journeyt... 25.07.2014

http://www.esa.int/Our_Activities/Human_Spaceflight/Research/The_worst_trip_aro und the_world 